PREPARED FOR THE U.S. DEPARTMENT OF ENERGY, UNDER CONTRACT DE-AC02-76CH03073

PPPL-3926

PPPL-3926

UC-70

Mini-conference and Related Sessions on Laboratory Plasma Astrophysics

by

Hantao Ji, Michael Brown, Scott C. Hsu, Hui Li, and R. Paul Drake

February 2004
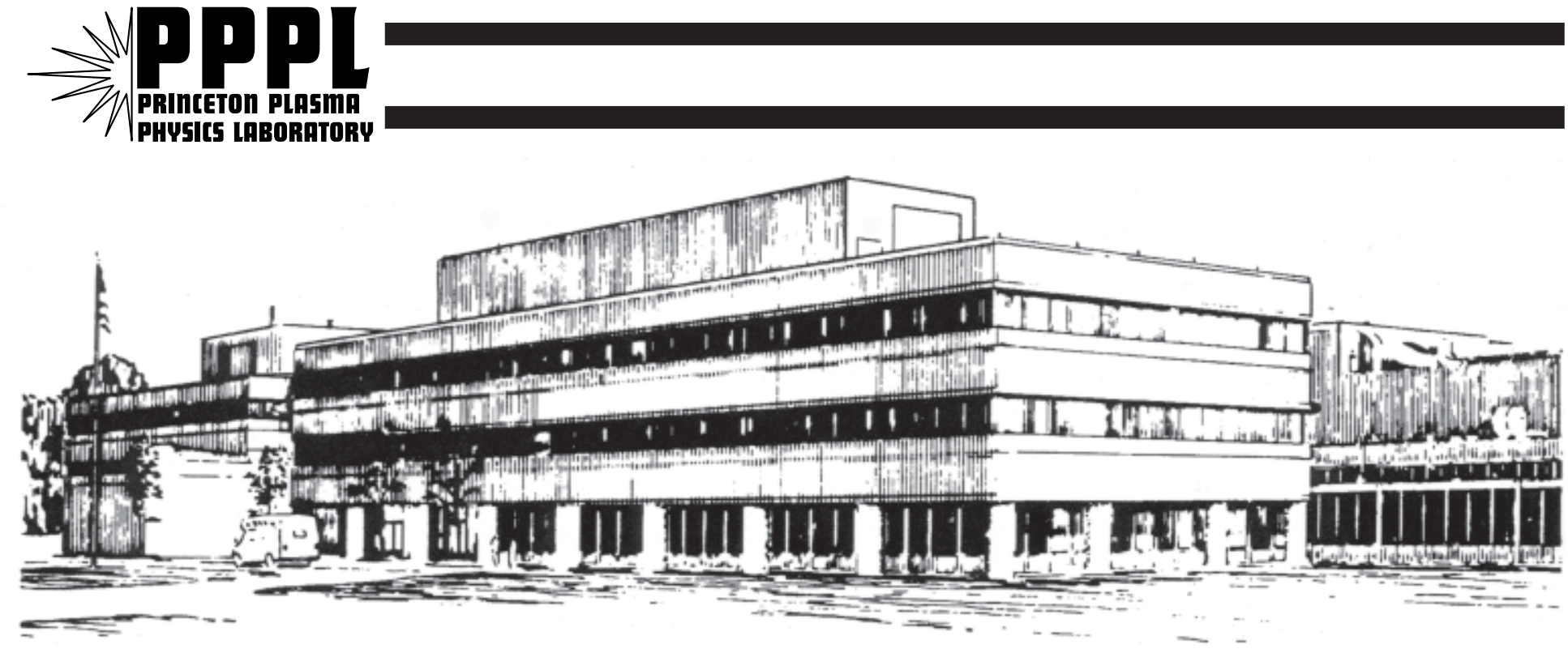

PRINCETON PLASMA PHYSICS LABORATORY PRINCETON UNIVERSITY, PRINCETON, NEW JERSEY 


\section{PPPL Reports Disclaimer}

This report was prepared as an account of work sponsored by an agency of the United States Government. Neither the United States Government nor any agency thereof, nor any of their employees, makes any warranty, express or implied, or assumes any legal liability or responsibility for the accuracy, completeness, or usefulness of any information, apparatus, product, or process disclosed, or represents that its use would not infringe privately owned rights. Reference herein to any specific commercial product, process, or service by trade name, trademark, manufacturer, or otherwise, does not necessarily constitute or imply its endorsement, recommendation, or favoring by the United States Government or any agency thereof. The views and opinions of authors expressed herein do not necessarily state or reflect those of the United States Government or any agency thereof.

\section{Availability}

This report is posted on the U.S. Department of Energy's Princeton Plasma Physics Laboratory Publications and Reports web site in Fiscal Year 2004. The home page for PPPL Reports and Publications is: http://www.pppl.gov/pub_report/

DOE and DOE Contractors can obtain copies of this report from:

U.S. Department of Energy

Office of Scientific and Technical Information

DOE Technical Information Services (DTIS)

P.O. Box 62

Oak Ridge, TN 37831

Telephone: (865) 576-8401

Fax: (865) 576-5728

Email: reports@adonis.osti.gov

This report is available to the general public from:

National Technical Information Service

U.S. Department of Commerce

5285 Port Royal Road

Springfield, VA 22161

Telephone: $1-800-553-6847$ or

(703) $605-6000$

Fax: (703) 321-8547

Internet: http://www.ntis.gov/ordering.htm 


\title{
Mini-conference and related sessions on laboratory plasma astrophysics
}

\author{
Hantao $\mathrm{Ji}^{*}$ \\ Center for Magnetic Self-organization in Astrophysical and Laboratory Plasmas \\ Princeton Plasma Physics Laboratory, \\ Princeton University, Princeton, New Jersey 08543 \\ Michael Brown \\ Center for Magnetic Self-organization in Astrophysical and Laboratory Plasmas \\ Department of Physics and Astronomy, \\ Swarthmore College, Swarthmore, Pennsylvania 19081 \\ Scott C. Hsu and Hui Li \\ Los Alamos National Laboratory, Los Alamos, New Mexico 87545 \\ R. Paul Drake \\ Department of Atmospheric, Oceanic and Space Science, \\ University of Michigan, Ann Arbor, Michigan 48109
}

(Dated: February 27, 2004)

\begin{abstract}
This paper provides a summary of some major physics issues and future perspectives discussed in the Mini-Conference on Laboratory Plasma Astrophysics. This Mini-conference, sponsored by the Topical Group on Plasma Astrophysics, was held as part of the American Physical Society's Division of Plasma Physics 2003 Annual Meeting (October 27-31, 2003). Also included are brief summaries of selected talks on the same topic presented at two invited paper sessions (including a tutorial) and two contributed focus oral sessions, which were organized in coordination with the Mini-Conference by the same organizers.

PACS numbers:
\end{abstract}




\section{INTRODUCTION}

There has been significant progress in the past few decades in both astrophysics and plasma physics. Advances in space physics and astrophysics are spearheaded by observations available from new satellites and ground-based observatories. In situ measurements of magnetospheric and solar wind plasmas, as well as high resolution images of solar and astrophysical plasmas at wavelengths ranging from radio to gamma rays, have revealed the properties and dynamics of space and astrophysical plasmas in great detail. Many of the observations have shown surprising phenomena and demand better understanding. At the same time, progress in the field of laboratory plasma physics has been led especially by advances in diagnostic techniques and the ability to perform realistic computer simulations to help understand experimental results. Therefore, there exist many opportunities to apply the knowledge gained in specially designed laboratory plasma experiments to help understand the dynamics of astrophysical plasmas. In fact, a wide range of experiments have been carried out recently with various laboratory devices to address astrophysical questions. These efforts have become collectively known as "laboratory plasma astrophysics."

In response to the interest expressed by both the astrophysics and plasma physics communities, an extended series of sessions including two invited sessions, two contributed focus oral sessions, and three Mini-conference sessions were organized on the topic of laboratory plasma astrophysics. The goal was to provide a platform to summarize and discuss recent progress in this area. At the end of each Mini-conference session, there was a half-hour panel discussion among the invited speakers and the audience to discuss opportunities and challenges within the topical area of that Mini-conference session. This paper intends to provide only brief summaries of selected contributions in these sessions including panel discussions without attempting to be a comprehensive review.

\section{INVITED SESSIONS}

The first presentation on the topic of laboratory plasma astrophysics was given by $\mathrm{R}$. Rosner of University of Chicago as a tutorial talk. The history of laboratory astrophysics including work outside of plasma physics was briefly reviewed. The earliest example started with Joseph Fraunhofer who accurately described "dark solar lines" in 1817. This was 
followed by a conjecture due to J. F. W. Hershel that these dark lines are results of absorption in cool terrestrial atmospheric gas or in the suns atmosphere. Later this was shown indeed to be true by R. Bunsen and G. Kirchhoff who did laboratory experiments on line absorption by cool gases. The significance of this work is best described by a quote from Sir William Huggins: "One important object of this original spectroscopic investigation of the light of the stars and other celestial bodies, namely to discover whether the same chemical elements as those of our earth are present throughout the universe, was most satisfactorily settled in the affirmative; a common chemistry, it was shown, exists throughout the universe." Later examples included measurements of extra-terrestrial magnetic fields, forbidden transitions, and dominance of hydrogen and helium in stars.

Then Rosner went into great detail in discussing a particular modern example of laboratory plasma astrophysics, the Rayleigh-Taylor instability during nuclear supernova explosions which signify the deaths of stars. ${ }^{1}$ The mixing efficiency between heavy fuels on top with light ashes on the bottom determines the nuclear burn rate and thus the macroscopic characteristics of a supernova. The mixing process, on the other hand, is described by details of the nonlinear evolution of the interface, and thus it is a microscopic process. Numerical simulations show that the mixing process is highly sensitive to various parameters including the number of simulation dimensions. The resultant mixing efficiency varies from fair agreement to complete disagreement with laboratory experiments. Close comparative studies based on both numerical simulations and laboratory experiments, a process termed "astroengineering" by Rosner, yielded detailed nonlinear physics knowledge of the Rayleigh-Taylor instability. Interestingly, however, the nuclear burn rates are rather robustly insensitive to the details of interface dynamics when nuclear reaction processes are incorporated into the calculation. This talk introduced a successful example of how closely coordinated laboratory experiments and numerical simulations can significantly deepen our understanding of astrophysical phenomena. It was pointed out that one of the roles of laboratory plasma astrophysics is to build our physical intuition for highly nonlinear astrophysical processes, even though the control parameters in the laboratory and in simulations may be very different than their astrophysical counterparts.

Further excellent examples on possible contributions from laboratory experiments to astrophysics were given by subsequent speakers. M. Yamada of the Princeton Plasma Physics Laboratory (PPPL) covered three topics under the theme of "magnetic self-organization," 
in light of a newly formed National Science Foundation (NSF) Physics Frontier Center. The first example concerns Taylor's theory ${ }^{2}$ to explain magnetic self-organization or relaxation phenomena observed in laboratory experiments, including reversed field pinches and spheromaks. With appropriate boundary conditions, minimizing magnetic energy while conserving magnetic helicity yields solutions for magnetic configurations strikingly similar to those observed in the laboratory. This idea is now being applied to astrophysics, solar coronal activity in particular, in attempts to explain sudden release of magnetic energy during solar flares. Despite the difficulties associated with boundary conditions, knowledge obtained in laboratory experiments is certainly impacting our understanding of astrophysical phenomena.

While Taylor's theory predicts the final states after relaxation, two important processes during the dynamics of relaxation are not described in detail. The first process concerns the dynamo effect, or spontaneous generation of (at least a part of) the magnetic field due to plasma dynamics. The " $\alpha$-effect," or electromotive electric field along the mean magnetic field due to fluctuations, has been successfully measured in detail in self-organized plasmas. ${ }^{3}$ While in general the measured $\alpha$-effect is in good agreement with MHD theories, laboratory studies provide some evidence that physics beyond magnetohydrodynamics (MHD), such as two-fluid effects, can be important under certain conditions. The second process concerns magnetic reconnection, or topological rearrangement of field lines in a conducting plasma resulting in efficient release of magnetic energy. Dedicated laboratory experiments on reconnection ${ }^{4}$ have provided a new testbed for numerous theories and simulations, and they have sometimes led to the discovery of new clues for mechanisms of fast reconnection. At present, forefront reconnection research focuses on physics beyond MHD in the reconnection region. Laboratory experiments contribute significantly to the understanding of these fundamental processes which play important roles in the dynamics of space and astrophysical plasmas.

A. Gailitis of the University of Latvia reported on the first experiment which successfully demonstrated dynamo action by motions of electrically conducting fluids by using liquid sodium. ${ }^{5}$ The group's persistence over more than two decades under both technical and financial constraints has finally paid off. Magnetic fields over $1 \mathrm{kG}$ are generated and sustained by liquid sodium motions, which are driven mechanically along a carefully designed path. Both the linear growth and nonlinear saturation of magnetic fields have been studied intensively. The only other group in the world which has successfully demonstrated dynamo 
action using liquid metals so far is located at Karlsruhe, Germany. ${ }^{6}$

R. P. Drake of the University of Michigan described recent progress in laboratory astrophysics at high energy density. Today's high-energy-density facilities enable one to produce pressures of many millions of atmospheres (Mbars), shock waves at hundreds of $\mathrm{km} / \mathrm{s}$, and temperatures of millions of degrees. These conditions permit the creation and study of both physical conditions and dynamical processes that occur in astrophysical systems. Measurements of the equation of state of hydrogen at pressures above 1 Mbar are directly relevant to gas giant planets such as Jupiter. Measurements of X-ray opacities and photoionized plasmas are key to the interpretation of astrophysical observations from Type Ia supernovae to black holes and neutron stars. Real uncertainties in astrophysical hydrodynamics can be addressed by well-scaled laboratory experiments, as is occuring today for example in experiments relevant to Rayleigh-Taylor instabilities in supernovae and to astrophysical jets. A newer forefront is the development of radiation hydrodynamic experiments. Ongoing work is seeking to produce and study radiatively collapsing shocks. The number of groups and experiments in this area is steadily increasing.

J. Egedal of the Massachusetts Institute of Technology (MIT) discussed motions of charged particles subject to a reconnecting electric field along an X-line with small guide field ( $B_{\text {guide }} \ll B_{\text {rec }}$ ). The measured electric current and potential in the Versatile Toroidal Facility (VTF) were found to be consistent with a particle trapping theory in this special magnetic topology. ${ }^{7}$ This phenomena was connected to Wind satellite observations of anisotropy in the magnetotail electron distribution function, ${ }^{8}$ providing a good example of how laboratory experimental results can improve understanding of space and astrophysical plasmas.

In a second invited session, J. Stone of Princeton University summarized state-of-the-art simulation studies of accretion disks. There has been rapid progress in this area of astrophysics since it was realized that efficient outward angular momentum transport could be explained by the magnetorotational instability (MRI). ${ }^{9}$ Simulations are now carried out globally rather than in a local box, and non-MHD effects such as ambipolar diffusion and radiation pressure are now included ${ }^{10}$. However, it was pointed out that it is difficult to compare these simulation and theoretical studies with reality due to the difficulties involved in measuring accretion disk magnetic fields. Laboratory studies of MRI, which are summarized later in this paper, would provide direct testbeds of these theories, especially the 
nonlinear effects.

The last talk in the second invited session was given by E. Wright of the University of California, Los Angeles (UCLA) on recent measurements of the cosmic microwave background from the early universe, using the WMAP (Wilkinson Microwave Anisotropy Probe) satellite. ${ }^{11}$ Sound waves in the primordial plasma (only 380,000 years after the big bang) were measured with unprecedented accuracy, leading to determinations of (1) the composition of the universe in terms of baryonic matter, dark matter, and dark energy, and (2) the time at which the universe re-ionized due to gravitational instability. A magnetic field of a few $\mathrm{mG}$ at recombination would produce detectable effects in the angular power spectrum and B-mode polarization that have not been seen. Therefore the magnetic pressure is at least a million times smaller than the cosmic microwave background radiation pressure. Although the physics of this primordial plasma is not necessarily the same as the usual plasma physics in the present universe, this talk provided an excellent opportunity for the DPP community to be exposed to a new frontier of plasma astrophysics at the far edge of modern cosmology.

\section{MINI-CONFERENCE SESSIONS}

\section{A. Mini-conference session I: high energy density experiments}

This session included work in nonlinear hydrodynamics, radiation hydrodynamics, equations of state, and extreme regimes of nuclear interactions. The session concluded with a panel discussion among speakers and audience, moderated by R. P. Drake of the University of Michigan. Nonlinear hydrodynamics has been the most active research topic in this area because such experiments are readily accessible on current facilities and are immediately relevant to state-of-the-art simulations. This last point was emphasized by V. Dwarkadas of the ASCI FLASH Center at the University of Chicago; he discussed the general problem of testing astrophysical simulations with laboratory experiments and some specific work to validate the FLASH code. A. Miles of the University of Maryland discussed experimentally motivated modeling, showing that the deep nonlinear evolution of the Rayleigh Taylor instability can be strongly affected by variations in initial conditions not unlike those anticipated in astrophysical systems. R. Williams of the Aldermaston Weapons Establishment in Britain discussed modeling of hydrodynamic flows that include clumps of denser material, as 
is the case when shock waves cross molecular clouds, for example. Experimental work in this area was discussed by J. Knauer of the Laboratory for Laser Energetics at the University of Rochester; he described the development of experiments relevant to astrophysical jets.

Recently there has been an increase in experiments focused on radiation hydrodynamic effects, reflecting increasing sophistication in the use of high-energy-density facilities. Two experimental investigations of radiative shocks were discussed. J. F. Hansen of Lawrence Livermore National Laboratory (LLNL) discussed spherical radiative shocks in low-density xenon gas, while A. Reighard of the University of Michigan discussed experiments to produce planar radiative shocks that collapse to high density. A longer-term area of focus has been the study of astrophysically relevant equations of state. In this area, Z pinches and lasers are extending work begun using gas guns. A. Mostovych of the Naval Research Laboratory (NRL) showed recent experimental work on the important problem of the hydrogen equation of state, supporting some degree of increased compressibility in the range of pressures in which hydrogen dissociates. S. Ichimaru of the University of Tokyo discussed theoretical studies of pycnonuclear fusion, which may produce greatly enhanced fusion rates under certain astrophysical conditions. In the panel discussion that closed the session, it was clear that there is also ongoing work of astrophysical relevance in radiative transfer even though none of this work was featured in this particular forum.

\section{B. Mini-conference session II: liquid metal and basic plasma experiments}

This session focused on laboratory experiments using liquid metals and basic plasma experiments (other than those used for fusion applications). There were three talks on liquid metal experiments, one talk on basic plasma experiments, and two talks on theoretical and numerical studies relevant to these experiments. The session concluded by a panel discussion among speakers and audience, moderated by H. Ji of PPPL.

D. Lathrop of the University of Maryland discussed results from a series of liquid sodium experiments at Maryland. In a recent experimental setup, an external magnetic field was applied along the rotation axis of an inner sphere. A stationary outer sphere allows the system to achieve spherical Couette flow. In general, torque on the inner sphere increases with increasing magnetic field. Sometimes a rotating mode with oscillations in both magnetic field and velocity was observed. It was commented that the increasing torque can 
be attributed to the magnetic field intercepting the highly conducting copper inner sphere, while the oscillations are possibly Alfvén waves traveling along a strong toroidal magnetic field generated by shearing. Although the claim that the oscillations are a form of MRI is highly debatable, the observations are certainly interesting and justify further investigations on MRI in spherical Couette flow geometry.

C. Forest of the University of Wisconsin discussed a large liquid sodium experiment (Madison Dynamo Experiment), with high velocities $\left(R_{\mathrm{m}}>50\right)$ and large size (1 m diameter), which should produce self generation of magnetic field. The key is that a particular flow field is required (counter-rotating toroidal flows in the northern/southern hemispheres with poloidal flows out of the north/south poles). The experimental design is based on kinematic modeling with prescribed flows. Since sodium and water have nearly identical hydrodynamic properties, water experiments were performed with laser velocimetry to demonstrate the required flow pattern. The physics goal is to study the nonlinear effects including back reaction on the flow due to the magnetic field.

F. Cattaneo of the University of Chicago discussed MHD turbulence in the small magnetic Prandtl number regime (the ratio of viscous to magnetic diffusion $P_{\mathrm{m}}=\nu / \eta=10^{-6}-10^{-5}$ ), where essentially all liquid metal experiments operate. Small $P_{\mathrm{m}}$ means that typical scales for $\boldsymbol{B}$ are much larger than that for $\boldsymbol{V}$. In other words, if a dynamo exists at all it must exist in the inertial range of the fluid turbulence. The dominant dissipation mechanism is via viscous diffusion and not resistive diffusion, and the fluid structure is "rough." This is important to study because if the saturation amplitude of $\boldsymbol{B}$ scales inversely with $P_{\mathrm{m}}$, then if $P_{\mathrm{m}} \ll 1$ the dynamo could operate but might saturate at trivial amplitudes. If $R_{\mathrm{m}}$ is large enough and $P_{\mathrm{m}}$ small enough, is there an exponentially growing dynamo with growth rate independent of dissipation? The results of this study suggest that the answer is yes.

S. Colgate of Los Alamos National Laboratory (LANL) discussed that, based on Faraday rotation measurements, up to $10^{60}$ ergs of magnetic energy are stored in $100 \mathrm{kpc}$ radio lobes ejected from galactic disks. This is comparable to the total gravitational potential energy released during the formation of the central super-massive black hole. A very efficient dynamo mechanism must exist, converting fluid energy to magnetic jets and plumes. The $\Omega$ effect converts poloidal to toroidal flux while the $\alpha$-effect due to star-disk collisions converts toroidal to poloidal flux. He discussed a cylindrical Couette flow liquid sodium experiment to simulate the physics. Plumes have been observed in a water experiment. MRI will also 
be studied during the first experimental phase to study $\Omega$-effect.

W. Gekelman of UCLA discussed upgraded LArge Plasma Device (LAPD-U) experiments on dense aluminum plasma expanding into a tenuous, magnetized plasma. A $150 \mathrm{MW}$ laser is used to ablate an aluminum rod immersed in an argon plasma $\left(B_{0}=1500 \mathrm{G}\right)$. An electron jet is generated along $B_{0}$ with background electrons providing the return current. This coaxial current structure launches a non-linear Alfvén wave packet that propagates down the magnetic field carried by ion polarization currents.

S. Cowley of UCLA discussed dynamo processes at large magnetic Prandtl numbers, applicable to the problem of galactic dynamo. Large $P_{\mathrm{m}}$ means that typical scales for $\boldsymbol{B}$ are much smaller than that for $\boldsymbol{V}$, opposite to the situation for liquid metals. Folding magnetic field structures embedded in very viscous plasmas are found to be typical in this case. Inverse cascade processes to transfer energy from this scale to larger scales were discussed, and proposed experiments by T. Carter (see below) on Alfvén wave cascades on LAPD-U were presented.

The concluding panel discussion focused on the relevance of laboratory plasmas to astrophysics. There was considerable discussion on the interplay among laboratory experiments, astrophysical observations, simulations, and theory. One can do direct comparisons of a general theory and a laboratory experiment (Lathrop). Since present day 3D simulations are limited to Reynolds numbers of 100-1000, they are inadequate to model astrophysical systems but are ideally suited to modeling and benchmarking laboratory experiments with Reynolds numbers of 100-1000 (Forest). At minimum, experiments can be used to "constrain the imagination of theorists." Even if the experiments only apply to limited regimes, constraining theory to "some regime is better than no regime" (Cattaneo). We need a dialog between laboratory experimentalists and astrophysicists. Experimentalists can "pick out one thing to study, we can't model the entire magnetosphere but we can model the interaction of two Alfvén waves" (Gekelman).

Mainstream astrophysicists are only now recognizing the importance of magnetic fields. "In order to understand the universe, you really need to understand MHD" (Cattaneo). Fluid turbulence has a long history and a standard model like Kolmogorov spectrum, but "no one knows the spectral index for inertial range turbulence in MHD" (Cowley). Theory goes faster than computation which goes faster than experiment (Colgate). We need to learn to expect the unexpected (Gekelman). 


\section{Mini-conference session III: magnetic self-organized plasma experiments}

This session focused on laboratory experiments on magnetic self-organized plasmas which are typically for fusion applications. It included three experimental talks and four related theoretical or numerical talks. The session concluded with a panel discussion among speakers and audience, moderated by M. Brown of Swarthmore College.

S. Prager of the University of Wiscosin discussed laboratory experiments on MST (Madison Symmetric Torus) relating to three key areas of astrophysical research: angular momentum transport, dynamo, and ion heating. Angular momentum must be lost in order for material to fall into the black hole at the center of accretion disks; the dominant mechanism is likely due to MRI. There is experimental evidence ${ }^{14}$ in MST that the plasma loses its momentum also through magnetic fluctuations but due to tearing modes instead of MRI. He presented data showing that when three waves were present, "braking" of plasma rotational velocity was observed. When only two waves were present, the rotational velocity grew. It was noted that effects of rotational shear on MHD stability were studied in an early theory ${ }^{12}$ in laboratory plasmas, similar but not identical to that in accretion disks. Representative measurements of dynamo effects and ion heating during self-organization were presented, followed by discussions on their mechanisms and relevance to astrophysics.

Y. Ono of the University of Tokyo discussed recent results from laboratory experiments on reconnection based on plasma merging. When the external driving force for reconnection is strong with a large guide field, magnetic flux piles up in the reconnection region with a relatively slow reconnection rate, and then the current sheet is mechanically ejected with a sudden increase in reconnection rate. The possible connections to coronal mass ejections (CME) on the sun were discussed.

H. Ji of PPPL presented new calculations by R. Kulsrud of PPPL on the anomalous resistivity and particle heating rates due to waves in the lower hybrid range of frequencies. This work was motivated by recent observations of electromagnetic waves in the current sheet of the Magnetic Reconnection Experiment (MRX). ${ }^{13}$ The effective resistivity due to waves with energy density of $\epsilon_{w}$ is given by

$$
\eta_{w} j=\frac{2 \gamma_{e}}{\omega} \frac{k \epsilon_{w}}{n e},
$$

where $\gamma_{e}$ is the total growth rate due to electrons (including nonlinear effect), $j$ the reconnecting current, $k$ the component of wavenumber vector along $j$, and $\omega$ the wave frequency. 
The total heating power due to waves are given by $\eta_{w} j^{2}$, which is split between electron and ion heating by ratios of $\left|V_{\phi}-V_{e}\right| /\left|V_{i}-V_{e}\right|$ and $\left|V_{\phi}-V_{i}\right| /\left|V_{i}-V_{e}\right|$. Here, $V_{\phi}, V_{i}$, and $V_{e}$ are wave phase velocity, ion and electron drift velocities, respectively.

P. Ricci of Torino, Italy, presented work from a collisionless PIC (particle-in-cell) reconnection code, starting with a Harris equilibrium and a $10 \%$ perturbation. He solves the Vlasov-Maxwell system with a uniform guide field $B_{0}$ and mass ratios of 25-180. The magnitude of $B_{0}$ adjusts the value of $\beta$. He finds an initial slow growth followed by fast reconnection. He observes the Hall quadrupolar structure in the reconnecting fields, but the structure vanishes at finite $B_{0}$, resulting in a slower reconnection rate.

S. Hsu of LANL showed a connection ${ }^{15}$ between an experiment with co-planar disk electrodes and an accretion disk. Both involve sheared rotation $(\boldsymbol{E} \times \boldsymbol{B}$ or Keplerian, respectively) and magnetic helicity injection. Distinct plasma morphologies result, depending on the parameter $\lambda=\mu_{0} I / \psi$, where $I$ is the electrode current and $\psi$ the vacuum magnetic flux intercepting the electrode. At low $\lambda$, he observes magnetic collimation of the discharge. At intermediate $\lambda$, he observes a kink in the column and a helical instability. At high $\lambda$, immediate detachment of the plasma from the gun with no column formation is observed. It can be shown that the Kruskal-Shafranov limit for MHD kink instability can be written as $\lambda>4 \pi / L_{c o l}$, and it was shown that the observed kinking occurred when the KruskalShafranov limit is exceeded. Furthermore, the kink is directly responsible for poloidal flux amplification and spheromak formation. ${ }^{16}$ He closed with a question: how is this related to the astrophysical observation of collimated jets? This work suggests that if $\lambda$ is too high, the column will kink and a spheromak will break off (which X. Tang dubs the "curse of the spheromak").

A. Bhattacharjee of the University of New Hampshire discussed the phenomenon of impulsive reconnection, which in the case of a solar flare, is characterized by a slow growth phase (30 minutes) in which flux piles up in the layer. This is followed by an explosive, non-linear, impulsive growth phase (1 minute) during which there is a sudden change in the time derivative of the growth rate. A disruption follows (10 seconds). He described a simulation that features adaptive mesh refinement (AMR).

D. Ryutov of LLNL described an astrophysical situation that could be simulated in the laboratory. Intense photoevaporation at the boundary of the Eagle nebula is thought to drive a Rayleigh-Taylor instability. The instability results in the observed fingering of the 
nebula. The role of magnetic fields was discussed; a field of $500 \mu \mathrm{G}$ parallel to the surface could explain the structure. This physics could be simulated in a laser-plasma experiment.

The concluding panel discussion focused on how to relate physics of laboratory plasmas to astrophysical plasmas. A typical difficulty is the difference in scales, and only in limited cases is it possible to scale experimental results to astrophysical scales (Ryutov). On the other hand, available 3D simulations are performed with similar scales, and experiments can provide valuable data to be compared and benchmarked, especially when little astrophysical data are available (Bhattacharjee). Well-designed experiments should be based on an interactive process between experimentalists and astrophysicists which simplifies complex astrophysical systems to elementary processes (Hsu). In addition to testing existing theories, sometimes laboratory experiments can introduce new ideas (Prager). It was also noted that in general the field of laboratory plasma astrophysics puts more burdens on the laboratory side rather than astrophysical side, but the situation may change if enough astrophysicists are attracted to this field because of its success. Other than the connections to astrophysics, of course, the laboratory plasma physics community needs its own applications, such as fusion and plasma processing, to maintain its own identity and thus stay viable (Ono).

\section{CONTRIBUTED FOCUS ORAL SESSIONS}

There were two focus oral sessions with 29 contributed talks on the topic of laboratory plasma astrophysics. The sessions featured talks from more than a dozen experiments around the world that are studying different physical processes relevant to astrophysics problems. Some of the issues discussed include: magnetic dissipation in the electron MHD regime, magnetic reconnection (both experiments and theory), liquid metal experiments for MRI and dynamo, magnetic self-organization phenomena, formation of solar flares and solar prominence, plasma wave generation and nonlinear wave interactions, and laser-driven plasma jets (both experiments and theory). Instead of summarizing each talk, summaries on each subcategory of experiments are given below as in the mini-conference: (a) high-energy-density experiments, (b) liquid metal and basic plasma experiments, and (c) magnetic self-organized plasma experiments. The summaries are not necessarily in the same order as the original talks. 


\section{A. High energy density experiments}

A. Poludnenko of the University of Rochester discussed mass-loaded flows through clumpy media relevant to astrophysical nebulae. This type of problem is also relevant in ICF laser experiments, where the foam in a target approximates the clumpy media. Such experiments can validate codes such as AstroBEAR. The subject to be explored is shock propagation versus time and as function of density distribution.

J. Grun of NRL discussed laboratory jets created by laser-driven hollow cones and wedges. The lab jets propagate into a low density foam or gas. This type of jet interaction with a background is thought to be similar to supernova jets. Recent developments in supernova research suggests that the SN explosion itself may be caused by the jet. The lab jets are $50-60 \mathrm{~km} / \mathrm{s}$. Scaling to astrophysics requires that the jet velocity is much greater than the internal sound speed.

S. Sublett of the University of Rochester discussed point-projection backlighting for laser experiments on OMEGA. She has achieved point projection of $3 \mathrm{~mm} \times 3 \mathrm{~mm}$ field of view and $10 \mu \mathrm{m}$ resolution. The technical challenge has been the size and spatial uniformity of the backlighter. She characterized the properties of $\mathrm{Cl}$, Ti, and Fe backlighters emitting in the 3-8 keV range. This tool is utilized to image both the jet and its interaction with a background medium.

S. Lebedev of Imperial College presented how radiatively cooled supersonic jets interact with a plasma environment which is dense or having a velocity (wind). B. Wilde of LANL presented numerical simulation results on modeling the radiative jet formation and propagation under various laser-driven conditions. Many widely used radiation-hydro codes have been used to compare with the experiments, which are very valuable in bench-marking the codes and in helping design new experiments. E. Liang of Rice University presented how an ultra-intense two-laser system can be used to create relativistic magnetized electron-positron plasmas and how the dynamics of these plasmas can be studied to address astrophysics problems. 


\section{B. Liquid metal and basic plasma experiments}

There were three talks on liquid metal experiments. H. Ji of PPPL discussed liquid gallium experiments to test MRI in a short Couette flow ${ }^{17}$. It is found that in a prototype water experiment the Ekman layer at the ends affects the rotation profile consistent with hydrodynamic simulations. Experimental modifications are being implemented to mitigate the effects of Ekman circulation for a clean demonstration of MRI and study of its efficiency for angular momentum transport. H. Beckley and R. Sonnenfeld at New Mexico Tech, in collab-

oration with S. Colgate of LANL, are studying Couette flow stability and the $\alpha-\Omega$ dynamo at high Reynolds number in a liquid sodium facility. Measurement of torque transmitted from inner to outer cylinder confirm the Ekman layer effect. The flow is hydrodynamically stable to Reynolds number of $4.4 \times 10^{6}$. The minimum level of background turbulence gives good hope of identifying both the $\Omega$ dynamo effect and MRI growth. Novel means of measuring magnetic field in the rotating system have been devised. Pressure sensors will also be utilized to detect MRI modes. R. Bayliss at Wisconsin has performed 3-D MHD simulations of the Madison Dynamo Experiment. The numerical results indicate saturated magnetic field energy at $10 \%$ of the flow energy, and the backreaction affects the flow profiles suggesting the importance of nonlinear effects. The dynamo occurs and saturates at $R_{\mathrm{m}}>120$.

The MRI will also be studied in plasma. Z. Wang of LANL reported on the new Flowing Magnetized Plasma (FMP) experiment. A coaxial gun produced plasma will be subject to $\boldsymbol{E} \times \boldsymbol{B}$ sheared rotation via biased electrodes and an externally imposed axial magnetic field. The goal is to establish a transient high $\beta$ rotational equilibrium in a regime where the MRI is expected to be linearly unstable, as indicated in numerical simulations by K. Noguchi, who gave a talk following Wang's. In contrast to liquid metal experiments, the magnetic Prandtl number can be varied in plasma, from approximately 0.1 to 10 in FMP. Thus, the MRI will be explored in varying and different regimes from the liquid metal experiments. The numerical work by Noguchi shows global, axisymmetric unstable MRI eigenmodes in a wide range of achievable FMP plasmas. MRI linear growth times are on the order of 100 microseconds compared to FMP plasma lifetimes of several milliseconds.

Several talks were related to magnetic dissipation processes including reconnection. R. Stenzel of UCLA presented experimental results on the stability and dissipation processes of a field-reversed configuration (FRC) in the electron MHD regime. He emphasized 
the role of 2D and 3D magnetic nulls in reconnection. He also showed evidence for preferential electron acceleration by these processes. S. Terry of PPPL presented MRX experimental results on high frequency electromagnetic fluctuations during reconnection. The fluctuation amplitude correlates with the enhanced reconnection rate, and fluctuations have been identified with the whistler waves. He also presented new results on detection of bifurcated current sheets during the early stage of driven reconnection. W. Daughton of LANL presented analytic and simulation studies of the lower-hybrid drift instability in a current sheet, ${ }^{18}$ and he found that modes with longer wavelengths penetrate deep into the current layer and have a significant electromagnetic component. This might have some bearing on the observed EM fluctuations in experiments. I. Furno of LANL presented data from the Reconnection Scaling Experiment (RSX) ${ }^{19}$ Preliminary results in the collisional regime and with strong guide field show that reconnection rate is consistent with the Sweet-Parker model. Further experiments are planned to study collisionless reconnection with varying guide field.

Another primary topic of basic plasma experiments was MHD phenomena and wave dynamics. P. Bellan of Caltech presented a mechanism ${ }^{20}$ on forming filamentary structures in MHD systems, motivated by Caltech experiments which show the tendency of uniform crosssectional area plasma columns to form. ${ }^{15,21} \mathrm{~S}$. Tripathi of Caltech presented experimental studies of solar prominences by merging dual-plasma arches. T. Carter of UCLA presented nonlinear interactions of shear Alfvén waves and wave-wave cascades with important implications for understanding MHD turbulence. S. Vincena of UCLA presented the dynamics of an expanding plasma, with results on associated instabilities and wave generation. W. Amatucci of NRL presented experimental results on sheared plasma flows driving broadband ion cyclotron waves and particle heating. E. Thomas of Auburn University also presented experimental results on ion cyclotron instability driven by flows and field-aligned currents. All these experiments have had varying degree of theory/simulation support, which is quite useful in both understanding the basic physics phenomena and validating the codes.

\section{Magnetic self-organized plasma experiments}

Both reconnection and dynamo have been studied during self-organization events in RFP plasmas in MST. J. Sarff of University of Wisconsin reported that the measured reconnection

layer widths around the reversal surface are about $5 \mathrm{~cm}$, somewhat smaller than the ion skin 
depth but larger than the ion gyroradius ${ }^{22}$. He also showed experimental evidence that this $m=0$ reconnection at the edge is driven nonlinearly by $m=1$ modes resonant at the core. G. Fiksel of University of Wisconsin presented new measurements of two-fluid dynamo effects, such as the Hall dynamo, which is more important than the MHD dynamo effect at certain locations. These results are consistent with some recent two-fluid theory on dynamo effects due to tearing instability.

M. Brown of Swarthmore College discussed the interplay between the Maxwell stress tensor governing electromagnetic fields and the Reynolds stress tensor (and/or the pressure tensor) governing flow fields and kinetic processes. Counter-helicity spheromak merging with oppositely directed fields generates self-organized structures (FRC) via magnetic reconnection. However, co-helicity merging with aligned fields generates self-organized structures (tilted spheromaks) without obvious reconnection.

E. Blackman of University of Rochester discussed the role of magnetic helicity conserving relaxation on dynamical time scales and its importance in astrophysics. ${ }^{23}$ It was emphasized that the dynamical evolution of various quantities can be described using nonlinear theories, and close comparisons with laboratory experiments can bridge the gap with astrophysical plasmas. He noted that flux "ribbons" (and not infinitesimal tubes) must be utilized to allow helicity conservation.

H. Li of LANL described relaxation processes in magnetic bubbles ejected from active galactic nuclei (AGN) as a key problem in the "magnetized universe." 24 He specifically talked about AGN outflows and how familiar plasma concepts such as helicity injection, helicity conservation, flux conversion, relaxation, and reconnection heating must play an important role in galaxy evolution and energetics. X. Tang, also of LANL, focused on a particular problem in the "magnetized universe" picture, that of quasar jet collimation. This process is envisioned to be similar to spheromak formation and DC helicity injection. The key scientific question is the flux conversion physics in the limit that injected toroidal flux is much larger than the background poloidal flux anchored in the accretion disk. He noted that this question can be addressed by laboratory experiments. 


\section{CONCLUSION}

Extensive coverage of "laboratory plasma astrophysics" was given in the 45th Annual APS-DPP meeting. Oral presentations alone totalled 57, including 7 invited talks, 21 miniconference talks, and 29 contributed talks. In addition, 3 panel discussion sessions were held to discuss opportunities and challenges within particular areas of this field. Although this coverage was not meant to be exhaustive, these sessions provided a rather comprehensive snapshot of the field at this time.

A few observations can be made. First, there are several "hot" topics and each motivates multiple experiments. To name a few: astrophysical hydrodynamics and radiation hydrodynamics (numerous experiments at large facilities), magnetic reconnection (at least 5 dedicated experiments), dynamo (at least 4 dedicated experiments), MRI (at least 3 dedicated experiments). Most of them involve magnetic field. We should also note that the number of hot topics is growing fast but only recently. Second, these experiments are typically operated by small groups with strong student participation. Often, these groups have a strong theoretical or numerical background both in plasma physics and in space physics or astrophysics. Third, the researchers are typically early in their careers, and this may be related to the fact that this field is only recently fast-growing. All these observations imply that this field will still be growing fast, at least in the near future.

A few caveats are in order to keep the field from overheating. First, posing the right question in experiments becomes increasingly important since the connection between laboratory and astrophysics is not always clear due to many factors, including scale separation and differences in boundary conditions. The problem one chooses to study should have clear astrophysical relevance, yet they must be clearly defined and testable in a laboratory experiment. One way to select problems can be based on a systematic process of breaking down complex astrophysical systems to elementary physics processes.

Second, close interplay with numerical simulations becomes increasingly viable for experiments to exist. In the foreseeable future, laboratory experiments have no hope of simulating the real scales of astrophysical systems. The same is true for numerical simulations, which nevertheless have been regarded as a major component of mainstream astrophysics research. Because both experiments and simulations are typically run with similar nondimensional parameters, experiments can be justified at the very least to provide benchmarking data for 
numerical simulations.

Here, the liquid metal experiments deserve some special remarks. Since liquid metals have well-controlled physical properties and their maintenance is easy (but could have difficulties in other aspects such as handling), these experiments represent a well-defined system for benchmarking MHD simulations. Their unique parameters, such as extremely small magnetic Prandtl numbers and incompressibility, provide a valuable regime, which is often relevant to astrophysics, for benchmarking astrophysical codes.

Finally, real impact from laboratory experiments on astrophysics still remains to be seen. Acceptance by the mainstream astrophysics community depends critically on how much laboratory experiments can contribute to our understanding of astrophysical phenomena. There is no doubt that laboratory experiments can be a testbed for many existing ideas and even provide a venue to discover new ideas, but at the same time the overall health and credibility of this growing field also depends critically on the rigor with which we interpret and apply experimental results.

\section{Acknowledgements}

This is supported by U.S. Department of Energy.

* Electronic address: hji@pppl.gov

1 A. Calder, B. Fryxell, T. Plewa, R. Rosner, L. Dursi, V. Weirs, T. Dupont, H. Robey, J. Kane, B. Remington, R.P. Drake, G. Dimonte, M. Zingale, F. Timmes, K. Olson, P. Ricker, P. MacNeice, and H. Tufo, Astrophys. J. Suppl. 143, 201 (2002).

2 J.B. Taylor, Rev. Mod. Phys. 58, 741 (1986).

3 H. Ji and S.C. Prager, Magnetohydrodynamics 18, 191 (2002).

4 M. Yamada, Earth Planets Space 53, 539 (2001).

5 A. Gailitis, Rev. Mod. Phys. 74, 973 (2002).

6 R. Stieglitz and U. Müller, Phys. Fluids 13, 561 (2001).

7 J. Egedal, A. Fasoli, and J. Nazemi, Phys. Rev. Lett. 90, 135003 (2003).

8 M. Øieroset, R. Lin, T. Phan, D. Larson, and S. Bale, Phys. Rev. Lett. 89, 195001 (2002). 
9 S. Balbus and J. Hawley, Rev. Mod. Phys. 70, 1 (1998).

10 T. Sano and J. Stone, Astrophys. J. 577, 534 (2000).

11 C. Bennett, M. Halpern, G. Hinshaw, N. Jarosik, A. Kogut, M. Limon, S. Meyer, L. Page, D. Spergel, G. Tucker, E. Wollack, E. Wright,C. Barnes, M. Greason, R. Hill, E. Komatsu, M. Nolta, N. Odegard, H. Peiris,L. Verde, and J. Weiland, Astrophys. J. Suppl. 148, 1 (2003).

12 A. Bondeson, and R. Iacono, and A. Bhattacharjee, Phys. Fluids 30, 2167 (1987).

13 H. Ji, S. Terry, M. Yamada, R. Kulsrud, A. Kuritsyn, and Y. Ren, "Electromagnetic Fluctuations during Fast Reconnection in a Laboratory Plasma", to be published in Phys. Rev. Lett. (2004).

14 A. K. Hansen, A. F. Almagri, D. Craig, D. J. Den Hartog, C. C. Hegna, S. C. Prager, and J. S. Sarff Phys. Rev. Lett. 85, 3408 (2000).

15 S. C. Hsu and P. M. Bellan, Mon. Not. R. Astron. Soc. 334, 257 (2002).

16 S. C. Hsu and P. M. Bellan, Phys. Rev. Lett. 90, 215002 (2003).

17 H. Ji, J. Goodman, and A. Kageyama, Mon. Not. R. Astron. Soc. 325, L1 (2001).

18 W. Daughton, Phys. Plasmas 10, 3103 (2003).

19 I. Furno et al., Rev. Sci. Instrum. 74, 2324 (2003).

20 P. M. Bellan, Phys. Plasmas 10, 1999 (2003).

21 J. F. Hansen and P. M. Bellan, Astrophys. J. 563, L183 (2001).

22 N. A. Crocker, G. Fiksel, S. C. Prager, and J. S. Sarff, Phys. Rev. Lett. 90, 035003 (2003).

23 E. G. Blackman, Phys. Rev. Lett. 89, 265007 (2002).

24 S. A. Colgate, H. Li, and V. Pariev, Phys. Plasmas 8, 2425 (2001). 


\title{
Mini-conference and related sessions on laboratory plasma astrophysics
}

\author{
Hantao $\mathrm{Ji}^{*}$ \\ Center for Magnetic Self-organization in Astrophysical and Laboratory Plasmas \\ Princeton Plasma Physics Laboratory, \\ Princeton University, Princeton, New Jersey 08543 \\ Michael Brown \\ Center for Magnetic Self-organization in Astrophysical and Laboratory Plasmas \\ Department of Physics and Astronomy, \\ Swarthmore College, Swarthmore, Pennsylvania 19081 \\ Scott C. Hsu and Hui Li \\ Los Alamos National Laboratory, Los Alamos, New Mexico 87545 \\ R. Paul Drake \\ Department of Atmospheric, Oceanic and Space Science, \\ University of Michigan, Ann Arbor, Michigan 48109
}

(Dated: February 27, 2004)

\begin{abstract}
This paper provides a summary of some major physics issues and future perspectives discussed in the Mini-Conference on Laboratory Plasma Astrophysics. This Mini-conference, sponsored by the Topical Group on Plasma Astrophysics, was held as part of the American Physical Society's Division of Plasma Physics 2003 Annual Meeting (October 27-31, 2003). Also included are brief summaries of selected talks on the same topic presented at two invited paper sessions (including a tutorial) and two contributed focus oral sessions, which were organized in coordination with the Mini-Conference by the same organizers.

PACS numbers:
\end{abstract}




\section{INTRODUCTION}

There has been significant progress in the past few decades in both astrophysics and plasma physics. Advances in space physics and astrophysics are spearheaded by observations available from new satellites and ground-based observatories. In situ measurements of magnetospheric and solar wind plasmas, as well as high resolution images of solar and astrophysical plasmas at wavelengths ranging from radio to gamma rays, have revealed the properties and dynamics of space and astrophysical plasmas in great detail. Many of the observations have shown surprising phenomena and demand better understanding. At the same time, progress in the field of laboratory plasma physics has been led especially by advances in diagnostic techniques and the ability to perform realistic computer simulations to help understand experimental results. Therefore, there exist many opportunities to apply the knowledge gained in specially designed laboratory plasma experiments to help understand the dynamics of astrophysical plasmas. In fact, a wide range of experiments have been carried out recently with various laboratory devices to address astrophysical questions. These efforts have become collectively known as "laboratory plasma astrophysics."

In response to the interest expressed by both the astrophysics and plasma physics communities, an extended series of sessions including two invited sessions, two contributed focus oral sessions, and three Mini-conference sessions were organized on the topic of laboratory plasma astrophysics. The goal was to provide a platform to summarize and discuss recent progress in this area. At the end of each Mini-conference session, there was a half-hour panel discussion among the invited speakers and the audience to discuss opportunities and challenges within the topical area of that Mini-conference session. This paper intends to provide only brief summaries of selected contributions in these sessions including panel discussions without attempting to be a comprehensive review.

\section{INVITED SESSIONS}

The first presentation on the topic of laboratory plasma astrophysics was given by $\mathrm{R}$. Rosner of University of Chicago as a tutorial talk. The history of laboratory astrophysics including work outside of plasma physics was briefly reviewed. The earliest example started with Joseph Fraunhofer who accurately described "dark solar lines" in 1817. This was 
followed by a conjecture due to J. F. W. Hershel that these dark lines are results of absorption in cool terrestrial atmospheric gas or in the suns atmosphere. Later this was shown indeed to be true by R. Bunsen and G. Kirchhoff who did laboratory experiments on line absorption by cool gases. The significance of this work is best described by a quote from Sir William Huggins: "One important object of this original spectroscopic investigation of the light of the stars and other celestial bodies, namely to discover whether the same chemical elements as those of our earth are present throughout the universe, was most satisfactorily settled in the affirmative; a common chemistry, it was shown, exists throughout the universe." Later examples included measurements of extra-terrestrial magnetic fields, forbidden transitions, and dominance of hydrogen and helium in stars.

Then Rosner went into great detail in discussing a particular modern example of laboratory plasma astrophysics, the Rayleigh-Taylor instability during nuclear supernova explosions which signify the deaths of stars. ${ }^{1}$ The mixing efficiency between heavy fuels on top with light ashes on the bottom determines the nuclear burn rate and thus the macroscopic characteristics of a supernova. The mixing process, on the other hand, is described by details of the nonlinear evolution of the interface, and thus it is a microscopic process. Numerical simulations show that the mixing process is highly sensitive to various parameters including the number of simulation dimensions. The resultant mixing efficiency varies from fair agreement to complete disagreement with laboratory experiments. Close comparative studies based on both numerical simulations and laboratory experiments, a process termed "astroengineering" by Rosner, yielded detailed nonlinear physics knowledge of the Rayleigh-Taylor instability. Interestingly, however, the nuclear burn rates are rather robustly insensitive to the details of interface dynamics when nuclear reaction processes are incorporated into the calculation. This talk introduced a successful example of how closely coordinated laboratory experiments and numerical simulations can significantly deepen our understanding of astrophysical phenomena. It was pointed out that one of the roles of laboratory plasma astrophysics is to build our physical intuition for highly nonlinear astrophysical processes, even though the control parameters in the laboratory and in simulations may be very different than their astrophysical counterparts.

Further excellent examples on possible contributions from laboratory experiments to astrophysics were given by subsequent speakers. M. Yamada of the Princeton Plasma Physics Laboratory (PPPL) covered three topics under the theme of "magnetic self-organization," 
in light of a newly formed National Science Foundation (NSF) Physics Frontier Center. The first example concerns Taylor's theory ${ }^{2}$ to explain magnetic self-organization or relaxation phenomena observed in laboratory experiments, including reversed field pinches and spheromaks. With appropriate boundary conditions, minimizing magnetic energy while conserving magnetic helicity yields solutions for magnetic configurations strikingly similar to those observed in the laboratory. This idea is now being applied to astrophysics, solar coronal activity in particular, in attempts to explain sudden release of magnetic energy during solar flares. Despite the difficulties associated with boundary conditions, knowledge obtained in laboratory experiments is certainly impacting our understanding of astrophysical phenomena.

While Taylor's theory predicts the final states after relaxation, two important processes during the dynamics of relaxation are not described in detail. The first process concerns the dynamo effect, or spontaneous generation of (at least a part of) the magnetic field due to plasma dynamics. The " $\alpha$-effect," or electromotive electric field along the mean magnetic field due to fluctuations, has been successfully measured in detail in self-organized plasmas. ${ }^{3}$ While in general the measured $\alpha$-effect is in good agreement with MHD theories, laboratory studies provide some evidence that physics beyond magnetohydrodynamics (MHD), such as two-fluid effects, can be important under certain conditions. The second process concerns magnetic reconnection, or topological rearrangement of field lines in a conducting plasma resulting in efficient release of magnetic energy. Dedicated laboratory experiments on reconnection ${ }^{4}$ have provided a new testbed for numerous theories and simulations, and they have sometimes led to the discovery of new clues for mechanisms of fast reconnection. At present, forefront reconnection research focuses on physics beyond MHD in the reconnection region. Laboratory experiments contribute significantly to the understanding of these fundamental processes which play important roles in the dynamics of space and astrophysical plasmas.

A. Gailitis of the University of Latvia reported on the first experiment which successfully demonstrated dynamo action by motions of electrically conducting fluids by using liquid sodium. ${ }^{5}$ The group's persistence over more than two decades under both technical and financial constraints has finally paid off. Magnetic fields over $1 \mathrm{kG}$ are generated and sustained by liquid sodium motions, which are driven mechanically along a carefully designed path. Both the linear growth and nonlinear saturation of magnetic fields have been studied intensively. The only other group in the world which has successfully demonstrated dynamo 
action using liquid metals so far is located at Karlsruhe, Germany. ${ }^{6}$

R. P. Drake of the University of Michigan described recent progress in laboratory astrophysics at high energy density. Today's high-energy-density facilities enable one to produce pressures of many millions of atmospheres (Mbars), shock waves at hundreds of $\mathrm{km} / \mathrm{s}$, and temperatures of millions of degrees. These conditions permit the creation and study of both physical conditions and dynamical processes that occur in astrophysical systems. Measurements of the equation of state of hydrogen at pressures above 1 Mbar are directly relevant to gas giant planets such as Jupiter. Measurements of X-ray opacities and photoionized plasmas are key to the interpretation of astrophysical observations from Type Ia supernovae to black holes and neutron stars. Real uncertainties in astrophysical hydrodynamics can be addressed by well-scaled laboratory experiments, as is occuring today for example in experiments relevant to Rayleigh-Taylor instabilities in supernovae and to astrophysical jets. A newer forefront is the development of radiation hydrodynamic experiments. Ongoing work is seeking to produce and study radiatively collapsing shocks. The number of groups and experiments in this area is steadily increasing.

J. Egedal of the Massachusetts Institute of Technology (MIT) discussed motions of charged particles subject to a reconnecting electric field along an X-line with small guide field ( $B_{\text {guide }} \ll B_{\text {rec }}$ ). The measured electric current and potential in the Versatile Toroidal Facility (VTF) were found to be consistent with a particle trapping theory in this special magnetic topology. ${ }^{7}$ This phenomena was connected to Wind satellite observations of anisotropy in the magnetotail electron distribution function, ${ }^{8}$ providing a good example of how laboratory experimental results can improve understanding of space and astrophysical plasmas.

In a second invited session, J. Stone of Princeton University summarized state-of-the-art simulation studies of accretion disks. There has been rapid progress in this area of astrophysics since it was realized that efficient outward angular momentum transport could be explained by the magnetorotational instability (MRI). ${ }^{9}$ Simulations are now carried out globally rather than in a local box, and non-MHD effects such as ambipolar diffusion and radiation pressure are now included ${ }^{10}$. However, it was pointed out that it is difficult to compare these simulation and theoretical studies with reality due to the difficulties involved in measuring accretion disk magnetic fields. Laboratory studies of MRI, which are summarized later in this paper, would provide direct testbeds of these theories, especially the 
nonlinear effects.

The last talk in the second invited session was given by E. Wright of the University of California, Los Angeles (UCLA) on recent measurements of the cosmic microwave background from the early universe, using the WMAP (Wilkinson Microwave Anisotropy Probe) satellite. ${ }^{11}$ Sound waves in the primordial plasma (only 380,000 years after the big bang) were measured with unprecedented accuracy, leading to determinations of (1) the composition of the universe in terms of baryonic matter, dark matter, and dark energy, and (2) the time at which the universe re-ionized due to gravitational instability. A magnetic field of a few $\mathrm{mG}$ at recombination would produce detectable effects in the angular power spectrum and B-mode polarization that have not been seen. Therefore the magnetic pressure is at least a million times smaller than the cosmic microwave background radiation pressure. Although the physics of this primordial plasma is not necessarily the same as the usual plasma physics in the present universe, this talk provided an excellent opportunity for the DPP community to be exposed to a new frontier of plasma astrophysics at the far edge of modern cosmology.

\section{MINI-CONFERENCE SESSIONS}

\section{A. Mini-conference session I: high energy density experiments}

This session included work in nonlinear hydrodynamics, radiation hydrodynamics, equations of state, and extreme regimes of nuclear interactions. The session concluded with a panel discussion among speakers and audience, moderated by R. P. Drake of the University of Michigan. Nonlinear hydrodynamics has been the most active research topic in this area because such experiments are readily accessible on current facilities and are immediately relevant to state-of-the-art simulations. This last point was emphasized by V. Dwarkadas of the ASCI FLASH Center at the University of Chicago; he discussed the general problem of testing astrophysical simulations with laboratory experiments and some specific work to validate the FLASH code. A. Miles of the University of Maryland discussed experimentally motivated modeling, showing that the deep nonlinear evolution of the Rayleigh Taylor instability can be strongly affected by variations in initial conditions not unlike those anticipated in astrophysical systems. R. Williams of the Aldermaston Weapons Establishment in Britain discussed modeling of hydrodynamic flows that include clumps of denser material, as 
is the case when shock waves cross molecular clouds, for example. Experimental work in this area was discussed by J. Knauer of the Laboratory for Laser Energetics at the University of Rochester; he described the development of experiments relevant to astrophysical jets.

Recently there has been an increase in experiments focused on radiation hydrodynamic effects, reflecting increasing sophistication in the use of high-energy-density facilities. Two experimental investigations of radiative shocks were discussed. J. F. Hansen of Lawrence Livermore National Laboratory (LLNL) discussed spherical radiative shocks in low-density xenon gas, while A. Reighard of the University of Michigan discussed experiments to produce planar radiative shocks that collapse to high density. A longer-term area of focus has been the study of astrophysically relevant equations of state. In this area, Z pinches and lasers are extending work begun using gas guns. A. Mostovych of the Naval Research Laboratory (NRL) showed recent experimental work on the important problem of the hydrogen equation of state, supporting some degree of increased compressibility in the range of pressures in which hydrogen dissociates. S. Ichimaru of the University of Tokyo discussed theoretical studies of pycnonuclear fusion, which may produce greatly enhanced fusion rates under certain astrophysical conditions. In the panel discussion that closed the session, it was clear that there is also ongoing work of astrophysical relevance in radiative transfer even though none of this work was featured in this particular forum.

\section{B. Mini-conference session II: liquid metal and basic plasma experiments}

This session focused on laboratory experiments using liquid metals and basic plasma experiments (other than those used for fusion applications). There were three talks on liquid metal experiments, one talk on basic plasma experiments, and two talks on theoretical and numerical studies relevant to these experiments. The session concluded by a panel discussion among speakers and audience, moderated by H. Ji of PPPL.

D. Lathrop of the University of Maryland discussed results from a series of liquid sodium experiments at Maryland. In a recent experimental setup, an external magnetic field was applied along the rotation axis of an inner sphere. A stationary outer sphere allows the system to achieve spherical Couette flow. In general, torque on the inner sphere increases with increasing magnetic field. Sometimes a rotating mode with oscillations in both magnetic field and velocity was observed. It was commented that the increasing torque can 
be attributed to the magnetic field intercepting the highly conducting copper inner sphere, while the oscillations are possibly Alfvén waves traveling along a strong toroidal magnetic field generated by shearing. Although the claim that the oscillations are a form of MRI is highly debatable, the observations are certainly interesting and justify further investigations on MRI in spherical Couette flow geometry.

C. Forest of the University of Wisconsin discussed a large liquid sodium experiment (Madison Dynamo Experiment), with high velocities $\left(R_{\mathrm{m}}>50\right)$ and large size (1 m diameter), which should produce self generation of magnetic field. The key is that a particular flow field is required (counter-rotating toroidal flows in the northern/southern hemispheres with poloidal flows out of the north/south poles). The experimental design is based on kinematic modeling with prescribed flows. Since sodium and water have nearly identical hydrodynamic properties, water experiments were performed with laser velocimetry to demonstrate the required flow pattern. The physics goal is to study the nonlinear effects including back reaction on the flow due to the magnetic field.

F. Cattaneo of the University of Chicago discussed MHD turbulence in the small magnetic Prandtl number regime (the ratio of viscous to magnetic diffusion $P_{\mathrm{m}}=\nu / \eta=10^{-6}-10^{-5}$ ), where essentially all liquid metal experiments operate. Small $P_{\mathrm{m}}$ means that typical scales for $\boldsymbol{B}$ are much larger than that for $\boldsymbol{V}$. In other words, if a dynamo exists at all it must exist in the inertial range of the fluid turbulence. The dominant dissipation mechanism is via viscous diffusion and not resistive diffusion, and the fluid structure is "rough." This is important to study because if the saturation amplitude of $\boldsymbol{B}$ scales inversely with $P_{\mathrm{m}}$, then if $P_{\mathrm{m}} \ll 1$ the dynamo could operate but might saturate at trivial amplitudes. If $R_{\mathrm{m}}$ is large enough and $P_{\mathrm{m}}$ small enough, is there an exponentially growing dynamo with growth rate independent of dissipation? The results of this study suggest that the answer is yes.

S. Colgate of Los Alamos National Laboratory (LANL) discussed that, based on Faraday rotation measurements, up to $10^{60}$ ergs of magnetic energy are stored in $100 \mathrm{kpc}$ radio lobes ejected from galactic disks. This is comparable to the total gravitational potential energy released during the formation of the central super-massive black hole. A very efficient dynamo mechanism must exist, converting fluid energy to magnetic jets and plumes. The $\Omega$ effect converts poloidal to toroidal flux while the $\alpha$-effect due to star-disk collisions converts toroidal to poloidal flux. He discussed a cylindrical Couette flow liquid sodium experiment to simulate the physics. Plumes have been observed in a water experiment. MRI will also 
be studied during the first experimental phase to study $\Omega$-effect.

W. Gekelman of UCLA discussed upgraded LArge Plasma Device (LAPD-U) experiments on dense aluminum plasma expanding into a tenuous, magnetized plasma. A $150 \mathrm{MW}$ laser is used to ablate an aluminum rod immersed in an argon plasma $\left(B_{0}=1500 \mathrm{G}\right)$. An electron jet is generated along $B_{0}$ with background electrons providing the return current. This coaxial current structure launches a non-linear Alfvén wave packet that propagates down the magnetic field carried by ion polarization currents.

S. Cowley of UCLA discussed dynamo processes at large magnetic Prandtl numbers, applicable to the problem of galactic dynamo. Large $P_{\mathrm{m}}$ means that typical scales for $\boldsymbol{B}$ are much smaller than that for $\boldsymbol{V}$, opposite to the situation for liquid metals. Folding magnetic field structures embedded in very viscous plasmas are found to be typical in this case. Inverse cascade processes to transfer energy from this scale to larger scales were discussed, and proposed experiments by T. Carter (see below) on Alfvén wave cascades on LAPD-U were presented.

The concluding panel discussion focused on the relevance of laboratory plasmas to astrophysics. There was considerable discussion on the interplay among laboratory experiments, astrophysical observations, simulations, and theory. One can do direct comparisons of a general theory and a laboratory experiment (Lathrop). Since present day 3D simulations are limited to Reynolds numbers of 100-1000, they are inadequate to model astrophysical systems but are ideally suited to modeling and benchmarking laboratory experiments with Reynolds numbers of 100-1000 (Forest). At minimum, experiments can be used to "constrain the imagination of theorists." Even if the experiments only apply to limited regimes, constraining theory to "some regime is better than no regime" (Cattaneo). We need a dialog between laboratory experimentalists and astrophysicists. Experimentalists can "pick out one thing to study, we can't model the entire magnetosphere but we can model the interaction of two Alfvén waves" (Gekelman).

Mainstream astrophysicists are only now recognizing the importance of magnetic fields. "In order to understand the universe, you really need to understand MHD" (Cattaneo). Fluid turbulence has a long history and a standard model like Kolmogorov spectrum, but "no one knows the spectral index for inertial range turbulence in MHD" (Cowley). Theory goes faster than computation which goes faster than experiment (Colgate). We need to learn to expect the unexpected (Gekelman). 


\section{Mini-conference session III: magnetic self-organized plasma experiments}

This session focused on laboratory experiments on magnetic self-organized plasmas which are typically for fusion applications. It included three experimental talks and four related theoretical or numerical talks. The session concluded with a panel discussion among speakers and audience, moderated by M. Brown of Swarthmore College.

S. Prager of the University of Wiscosin discussed laboratory experiments on MST (Madison Symmetric Torus) relating to three key areas of astrophysical research: angular momentum transport, dynamo, and ion heating. Angular momentum must be lost in order for material to fall into the black hole at the center of accretion disks; the dominant mechanism is likely due to MRI. There is experimental evidence ${ }^{14}$ in MST that the plasma loses its momentum also through magnetic fluctuations but due to tearing modes instead of MRI. He presented data showing that when three waves were present, "braking" of plasma rotational velocity was observed. When only two waves were present, the rotational velocity grew. It was noted that effects of rotational shear on MHD stability were studied in an early theory ${ }^{12}$ in laboratory plasmas, similar but not identical to that in accretion disks. Representative measurements of dynamo effects and ion heating during self-organization were presented, followed by discussions on their mechanisms and relevance to astrophysics.

Y. Ono of the University of Tokyo discussed recent results from laboratory experiments on reconnection based on plasma merging. When the external driving force for reconnection is strong with a large guide field, magnetic flux piles up in the reconnection region with a relatively slow reconnection rate, and then the current sheet is mechanically ejected with a sudden increase in reconnection rate. The possible connections to coronal mass ejections (CME) on the sun were discussed.

H. Ji of PPPL presented new calculations by R. Kulsrud of PPPL on the anomalous resistivity and particle heating rates due to waves in the lower hybrid range of frequencies. This work was motivated by recent observations of electromagnetic waves in the current sheet of the Magnetic Reconnection Experiment (MRX). ${ }^{13}$ The effective resistivity due to waves with energy density of $\epsilon_{w}$ is given by

$$
\eta_{w} j=\frac{2 \gamma_{e}}{\omega} \frac{k \epsilon_{w}}{n e},
$$

where $\gamma_{e}$ is the total growth rate due to electrons (including nonlinear effect), $j$ the reconnecting current, $k$ the component of wavenumber vector along $j$, and $\omega$ the wave frequency. 
The total heating power due to waves are given by $\eta_{w} j^{2}$, which is split between electron and ion heating by ratios of $\left|V_{\phi}-V_{e}\right| /\left|V_{i}-V_{e}\right|$ and $\left|V_{\phi}-V_{i}\right| /\left|V_{i}-V_{e}\right|$. Here, $V_{\phi}, V_{i}$, and $V_{e}$ are wave phase velocity, ion and electron drift velocities, respectively.

P. Ricci of Torino, Italy, presented work from a collisionless PIC (particle-in-cell) reconnection code, starting with a Harris equilibrium and a $10 \%$ perturbation. He solves the Vlasov-Maxwell system with a uniform guide field $B_{0}$ and mass ratios of 25-180. The magnitude of $B_{0}$ adjusts the value of $\beta$. He finds an initial slow growth followed by fast reconnection. He observes the Hall quadrupolar structure in the reconnecting fields, but the structure vanishes at finite $B_{0}$, resulting in a slower reconnection rate.

S. Hsu of LANL showed a connection ${ }^{15}$ between an experiment with co-planar disk electrodes and an accretion disk. Both involve sheared rotation $(\boldsymbol{E} \times \boldsymbol{B}$ or Keplerian, respectively) and magnetic helicity injection. Distinct plasma morphologies result, depending on the parameter $\lambda=\mu_{0} I / \psi$, where $I$ is the electrode current and $\psi$ the vacuum magnetic flux intercepting the electrode. At low $\lambda$, he observes magnetic collimation of the discharge. At intermediate $\lambda$, he observes a kink in the column and a helical instability. At high $\lambda$, immediate detachment of the plasma from the gun with no column formation is observed. It can be shown that the Kruskal-Shafranov limit for MHD kink instability can be written as $\lambda>4 \pi / L_{c o l}$, and it was shown that the observed kinking occurred when the KruskalShafranov limit is exceeded. Furthermore, the kink is directly responsible for poloidal flux amplification and spheromak formation. ${ }^{16}$ He closed with a question: how is this related to the astrophysical observation of collimated jets? This work suggests that if $\lambda$ is too high, the column will kink and a spheromak will break off (which X. Tang dubs the "curse of the spheromak").

A. Bhattacharjee of the University of New Hampshire discussed the phenomenon of impulsive reconnection, which in the case of a solar flare, is characterized by a slow growth phase (30 minutes) in which flux piles up in the layer. This is followed by an explosive, non-linear, impulsive growth phase (1 minute) during which there is a sudden change in the time derivative of the growth rate. A disruption follows (10 seconds). He described a simulation that features adaptive mesh refinement (AMR).

D. Ryutov of LLNL described an astrophysical situation that could be simulated in the laboratory. Intense photoevaporation at the boundary of the Eagle nebula is thought to drive a Rayleigh-Taylor instability. The instability results in the observed fingering of the 
nebula. The role of magnetic fields was discussed; a field of $500 \mu \mathrm{G}$ parallel to the surface could explain the structure. This physics could be simulated in a laser-plasma experiment.

The concluding panel discussion focused on how to relate physics of laboratory plasmas to astrophysical plasmas. A typical difficulty is the difference in scales, and only in limited cases is it possible to scale experimental results to astrophysical scales (Ryutov). On the other hand, available 3D simulations are performed with similar scales, and experiments can provide valuable data to be compared and benchmarked, especially when little astrophysical data are available (Bhattacharjee). Well-designed experiments should be based on an interactive process between experimentalists and astrophysicists which simplifies complex astrophysical systems to elementary processes (Hsu). In addition to testing existing theories, sometimes laboratory experiments can introduce new ideas (Prager). It was also noted that in general the field of laboratory plasma astrophysics puts more burdens on the laboratory side rather than astrophysical side, but the situation may change if enough astrophysicists are attracted to this field because of its success. Other than the connections to astrophysics, of course, the laboratory plasma physics community needs its own applications, such as fusion and plasma processing, to maintain its own identity and thus stay viable (Ono).

\section{CONTRIBUTED FOCUS ORAL SESSIONS}

There were two focus oral sessions with 29 contributed talks on the topic of laboratory plasma astrophysics. The sessions featured talks from more than a dozen experiments around the world that are studying different physical processes relevant to astrophysics problems. Some of the issues discussed include: magnetic dissipation in the electron MHD regime, magnetic reconnection (both experiments and theory), liquid metal experiments for MRI and dynamo, magnetic self-organization phenomena, formation of solar flares and solar prominence, plasma wave generation and nonlinear wave interactions, and laser-driven plasma jets (both experiments and theory). Instead of summarizing each talk, summaries on each subcategory of experiments are given below as in the mini-conference: (a) high-energy-density experiments, (b) liquid metal and basic plasma experiments, and (c) magnetic self-organized plasma experiments. The summaries are not necessarily in the same order as the original talks. 


\section{A. High energy density experiments}

A. Poludnenko of the University of Rochester discussed mass-loaded flows through clumpy media relevant to astrophysical nebulae. This type of problem is also relevant in ICF laser experiments, where the foam in a target approximates the clumpy media. Such experiments can validate codes such as AstroBEAR. The subject to be explored is shock propagation versus time and as function of density distribution.

J. Grun of NRL discussed laboratory jets created by laser-driven hollow cones and wedges. The lab jets propagate into a low density foam or gas. This type of jet interaction with a background is thought to be similar to supernova jets. Recent developments in supernova research suggests that the SN explosion itself may be caused by the jet. The lab jets are $50-60 \mathrm{~km} / \mathrm{s}$. Scaling to astrophysics requires that the jet velocity is much greater than the internal sound speed.

S. Sublett of the University of Rochester discussed point-projection backlighting for laser experiments on OMEGA. She has achieved point projection of $3 \mathrm{~mm} \times 3 \mathrm{~mm}$ field of view and $10 \mu \mathrm{m}$ resolution. The technical challenge has been the size and spatial uniformity of the backlighter. She characterized the properties of $\mathrm{Cl}$, Ti, and Fe backlighters emitting in the 3-8 keV range. This tool is utilized to image both the jet and its interaction with a background medium.

S. Lebedev of Imperial College presented how radiatively cooled supersonic jets interact with a plasma environment which is dense or having a velocity (wind). B. Wilde of LANL presented numerical simulation results on modeling the radiative jet formation and propagation under various laser-driven conditions. Many widely used radiation-hydro codes have been used to compare with the experiments, which are very valuable in bench-marking the codes and in helping design new experiments. E. Liang of Rice University presented how an ultra-intense two-laser system can be used to create relativistic magnetized electron-positron plasmas and how the dynamics of these plasmas can be studied to address astrophysics problems. 


\section{B. Liquid metal and basic plasma experiments}

There were three talks on liquid metal experiments. H. Ji of PPPL discussed liquid gallium experiments to test MRI in a short Couette flow ${ }^{17}$. It is found that in a prototype water experiment the Ekman layer at the ends affects the rotation profile consistent with hydrodynamic simulations. Experimental modifications are being implemented to mitigate the effects of Ekman circulation for a clean demonstration of MRI and study of its efficiency for angular momentum transport. H. Beckley and R. Sonnenfeld at New Mexico Tech, in collab-

oration with S. Colgate of LANL, are studying Couette flow stability and the $\alpha-\Omega$ dynamo at high Reynolds number in a liquid sodium facility. Measurement of torque transmitted from inner to outer cylinder confirm the Ekman layer effect. The flow is hydrodynamically stable to Reynolds number of $4.4 \times 10^{6}$. The minimum level of background turbulence gives good hope of identifying both the $\Omega$ dynamo effect and MRI growth. Novel means of measuring magnetic field in the rotating system have been devised. Pressure sensors will also be utilized to detect MRI modes. R. Bayliss at Wisconsin has performed 3-D MHD simulations of the Madison Dynamo Experiment. The numerical results indicate saturated magnetic field energy at $10 \%$ of the flow energy, and the backreaction affects the flow profiles suggesting the importance of nonlinear effects. The dynamo occurs and saturates at $R_{\mathrm{m}}>120$.

The MRI will also be studied in plasma. Z. Wang of LANL reported on the new Flowing Magnetized Plasma (FMP) experiment. A coaxial gun produced plasma will be subject to $\boldsymbol{E} \times \boldsymbol{B}$ sheared rotation via biased electrodes and an externally imposed axial magnetic field. The goal is to establish a transient high $\beta$ rotational equilibrium in a regime where the MRI is expected to be linearly unstable, as indicated in numerical simulations by K. Noguchi, who gave a talk following Wang's. In contrast to liquid metal experiments, the magnetic Prandtl number can be varied in plasma, from approximately 0.1 to 10 in FMP. Thus, the MRI will be explored in varying and different regimes from the liquid metal experiments. The numerical work by Noguchi shows global, axisymmetric unstable MRI eigenmodes in a wide range of achievable FMP plasmas. MRI linear growth times are on the order of 100 microseconds compared to FMP plasma lifetimes of several milliseconds.

Several talks were related to magnetic dissipation processes including reconnection. R. Stenzel of UCLA presented experimental results on the stability and dissipation processes of a field-reversed configuration (FRC) in the electron MHD regime. He emphasized 
the role of 2D and 3D magnetic nulls in reconnection. He also showed evidence for preferential electron acceleration by these processes. S. Terry of PPPL presented MRX experimental results on high frequency electromagnetic fluctuations during reconnection. The fluctuation amplitude correlates with the enhanced reconnection rate, and fluctuations have been identified with the whistler waves. He also presented new results on detection of bifurcated current sheets during the early stage of driven reconnection. W. Daughton of LANL presented analytic and simulation studies of the lower-hybrid drift instability in a current sheet, ${ }^{18}$ and he found that modes with longer wavelengths penetrate deep into the current layer and have a significant electromagnetic component. This might have some bearing on the observed EM fluctuations in experiments. I. Furno of LANL presented data from the Reconnection Scaling Experiment (RSX) ${ }^{19}$ Preliminary results in the collisional regime and with strong guide field show that reconnection rate is consistent with the Sweet-Parker model. Further experiments are planned to study collisionless reconnection with varying guide field.

Another primary topic of basic plasma experiments was MHD phenomena and wave dynamics. P. Bellan of Caltech presented a mechanism ${ }^{20}$ on forming filamentary structures in MHD systems, motivated by Caltech experiments which show the tendency of uniform crosssectional area plasma columns to form. ${ }^{15,21} \mathrm{~S}$. Tripathi of Caltech presented experimental studies of solar prominences by merging dual-plasma arches. T. Carter of UCLA presented nonlinear interactions of shear Alfvén waves and wave-wave cascades with important implications for understanding MHD turbulence. S. Vincena of UCLA presented the dynamics of an expanding plasma, with results on associated instabilities and wave generation. W. Amatucci of NRL presented experimental results on sheared plasma flows driving broadband ion cyclotron waves and particle heating. E. Thomas of Auburn University also presented experimental results on ion cyclotron instability driven by flows and field-aligned currents. All these experiments have had varying degree of theory/simulation support, which is quite useful in both understanding the basic physics phenomena and validating the codes.

\section{Magnetic self-organized plasma experiments}

Both reconnection and dynamo have been studied during self-organization events in RFP plasmas in MST. J. Sarff of University of Wisconsin reported that the measured reconnection

layer widths around the reversal surface are about $5 \mathrm{~cm}$, somewhat smaller than the ion skin 
depth but larger than the ion gyroradius ${ }^{22}$. He also showed experimental evidence that this $m=0$ reconnection at the edge is driven nonlinearly by $m=1$ modes resonant at the core. G. Fiksel of University of Wisconsin presented new measurements of two-fluid dynamo effects, such as the Hall dynamo, which is more important than the MHD dynamo effect at certain locations. These results are consistent with some recent two-fluid theory on dynamo effects due to tearing instability.

M. Brown of Swarthmore College discussed the interplay between the Maxwell stress tensor governing electromagnetic fields and the Reynolds stress tensor (and/or the pressure tensor) governing flow fields and kinetic processes. Counter-helicity spheromak merging with oppositely directed fields generates self-organized structures (FRC) via magnetic reconnection. However, co-helicity merging with aligned fields generates self-organized structures (tilted spheromaks) without obvious reconnection.

E. Blackman of University of Rochester discussed the role of magnetic helicity conserving relaxation on dynamical time scales and its importance in astrophysics. ${ }^{23}$ It was emphasized that the dynamical evolution of various quantities can be described using nonlinear theories, and close comparisons with laboratory experiments can bridge the gap with astrophysical plasmas. He noted that flux "ribbons" (and not infinitesimal tubes) must be utilized to allow helicity conservation.

H. Li of LANL described relaxation processes in magnetic bubbles ejected from active galactic nuclei (AGN) as a key problem in the "magnetized universe." 24 He specifically talked about AGN outflows and how familiar plasma concepts such as helicity injection, helicity conservation, flux conversion, relaxation, and reconnection heating must play an important role in galaxy evolution and energetics. X. Tang, also of LANL, focused on a particular problem in the "magnetized universe" picture, that of quasar jet collimation. This process is envisioned to be similar to spheromak formation and DC helicity injection. The key scientific question is the flux conversion physics in the limit that injected toroidal flux is much larger than the background poloidal flux anchored in the accretion disk. He noted that this question can be addressed by laboratory experiments. 


\section{CONCLUSION}

Extensive coverage of "laboratory plasma astrophysics" was given in the 45th Annual APS-DPP meeting. Oral presentations alone totalled 57, including 7 invited talks, 21 miniconference talks, and 29 contributed talks. In addition, 3 panel discussion sessions were held to discuss opportunities and challenges within particular areas of this field. Although this coverage was not meant to be exhaustive, these sessions provided a rather comprehensive snapshot of the field at this time.

A few observations can be made. First, there are several "hot" topics and each motivates multiple experiments. To name a few: astrophysical hydrodynamics and radiation hydrodynamics (numerous experiments at large facilities), magnetic reconnection (at least 5 dedicated experiments), dynamo (at least 4 dedicated experiments), MRI (at least 3 dedicated experiments). Most of them involve magnetic field. We should also note that the number of hot topics is growing fast but only recently. Second, these experiments are typically operated by small groups with strong student participation. Often, these groups have a strong theoretical or numerical background both in plasma physics and in space physics or astrophysics. Third, the researchers are typically early in their careers, and this may be related to the fact that this field is only recently fast-growing. All these observations imply that this field will still be growing fast, at least in the near future.

A few caveats are in order to keep the field from overheating. First, posing the right question in experiments becomes increasingly important since the connection between laboratory and astrophysics is not always clear due to many factors, including scale separation and differences in boundary conditions. The problem one chooses to study should have clear astrophysical relevance, yet they must be clearly defined and testable in a laboratory experiment. One way to select problems can be based on a systematic process of breaking down complex astrophysical systems to elementary physics processes.

Second, close interplay with numerical simulations becomes increasingly viable for experiments to exist. In the foreseeable future, laboratory experiments have no hope of simulating the real scales of astrophysical systems. The same is true for numerical simulations, which nevertheless have been regarded as a major component of mainstream astrophysics research. Because both experiments and simulations are typically run with similar nondimensional parameters, experiments can be justified at the very least to provide benchmarking data for 
numerical simulations.

Here, the liquid metal experiments deserve some special remarks. Since liquid metals have well-controlled physical properties and their maintenance is easy (but could have difficulties in other aspects such as handling), these experiments represent a well-defined system for benchmarking MHD simulations. Their unique parameters, such as extremely small magnetic Prandtl numbers and incompressibility, provide a valuable regime, which is often relevant to astrophysics, for benchmarking astrophysical codes.

Finally, real impact from laboratory experiments on astrophysics still remains to be seen. Acceptance by the mainstream astrophysics community depends critically on how much laboratory experiments can contribute to our understanding of astrophysical phenomena. There is no doubt that laboratory experiments can be a testbed for many existing ideas and even provide a venue to discover new ideas, but at the same time the overall health and credibility of this growing field also depends critically on the rigor with which we interpret and apply experimental results.

\section{Acknowledgements}

This is supported by U.S. Department of Energy.

* Electronic address: hji@pppl.gov

1 A. Calder, B. Fryxell, T. Plewa, R. Rosner, L. Dursi, V. Weirs, T. Dupont, H. Robey, J. Kane, B. Remington, R.P. Drake, G. Dimonte, M. Zingale, F. Timmes, K. Olson, P. Ricker, P. MacNeice, and H. Tufo, Astrophys. J. Suppl. 143, 201 (2002).

2 J.B. Taylor, Rev. Mod. Phys. 58, 741 (1986).

3 H. Ji and S.C. Prager, Magnetohydrodynamics 18, 191 (2002).

4 M. Yamada, Earth Planets Space 53, 539 (2001).

5 A. Gailitis, Rev. Mod. Phys. 74, 973 (2002).

6 R. Stieglitz and U. Müller, Phys. Fluids 13, 561 (2001).

7 J. Egedal, A. Fasoli, and J. Nazemi, Phys. Rev. Lett. 90, 135003 (2003).

8 M. Øieroset, R. Lin, T. Phan, D. Larson, and S. Bale, Phys. Rev. Lett. 89, 195001 (2002). 
9 S. Balbus and J. Hawley, Rev. Mod. Phys. 70, 1 (1998).

10 T. Sano and J. Stone, Astrophys. J. 577, 534 (2000).

11 C. Bennett, M. Halpern, G. Hinshaw, N. Jarosik, A. Kogut, M. Limon, S. Meyer, L. Page, D. Spergel, G. Tucker, E. Wollack, E. Wright,C. Barnes, M. Greason, R. Hill, E. Komatsu, M. Nolta, N. Odegard, H. Peiris,L. Verde, and J. Weiland, Astrophys. J. Suppl. 148, 1 (2003).

12 A. Bondeson, and R. Iacono, and A. Bhattacharjee, Phys. Fluids 30, 2167 (1987).

13 H. Ji, S. Terry, M. Yamada, R. Kulsrud, A. Kuritsyn, and Y. Ren, "Electromagnetic Fluctuations during Fast Reconnection in a Laboratory Plasma", to be published in Phys. Rev. Lett. (2004).

14 A. K. Hansen, A. F. Almagri, D. Craig, D. J. Den Hartog, C. C. Hegna, S. C. Prager, and J. S. Sarff Phys. Rev. Lett. 85, 3408 (2000).

15 S. C. Hsu and P. M. Bellan, Mon. Not. R. Astron. Soc. 334, 257 (2002).

16 S. C. Hsu and P. M. Bellan, Phys. Rev. Lett. 90, 215002 (2003).

17 H. Ji, J. Goodman, and A. Kageyama, Mon. Not. R. Astron. Soc. 325, L1 (2001).

18 W. Daughton, Phys. Plasmas 10, 3103 (2003).

19 I. Furno et al., Rev. Sci. Instrum. 74, 2324 (2003).

20 P. M. Bellan, Phys. Plasmas 10, 1999 (2003).

21 J. F. Hansen and P. M. Bellan, Astrophys. J. 563, L183 (2001).

22 N. A. Crocker, G. Fiksel, S. C. Prager, and J. S. Sarff, Phys. Rev. Lett. 90, 035003 (2003).

23 E. G. Blackman, Phys. Rev. Lett. 89, 265007 (2002).

24 S. A. Colgate, H. Li, and V. Pariev, Phys. Plasmas 8, 2425 (2001). 


\section{External Distribution}

Plasma Research Laboratory, Australian National University, Australia

Professor I.R. Jones, Flinders University, Australia

Professor João Canalle, Instituto de Fisica DEQ/IF - UERJ, Brazil

Mr. Gerson O. Ludwig, Instituto Nacional de Pesquisas, Brazil

Dr. P.H. Sakanaka, Instituto Fisica, Brazil

The Librarian, Culham Laboratory, England

Mrs. S.A. Hutchinson, JET Library, England

Professor M.N. Bussac, Ecole Polytechnique, France

Librarian, Max-Planck-Institut für Plasmaphysik, Germany

Jolan Moldvai, Reports Library, Hungarian Academy of Sciences, Central Research Institute for Physics, Hungary

Dr. P. Kaw, Institute for Plasma Research, India

Ms. P.J. Pathak, Librarian, Institute for Plasma Research, India

Ms. Clelia De Palo, Associazione EURATOM-ENEA, Italy

Dr. G. Grosso, Instituto di Fisica del Plasma, Italy

Librarian, Naka Fusion Research Establishment, JAERI, Japan

Library, Laboratory for Complex Energy Processes, Institute for Advanced Study, Kyoto University, Japan

Research Information Center, National Institute for Fusion Science, Japan

Dr. O. Mitarai, Kyushu Tokai University, Japan

Dr. Jiangang Li, Institute of Plasma Physics, Chinese Academy of Sciences, People's Republic of China

Professor Yuping Huo, School of Physical Science and Technology, People's Republic of China

Library, Academia Sinica, Institute of Plasma Physics, People's Republic of China

Librarian, Institute of Physics, Chinese Academy of Sciences, People's Republic of China

Dr. S. Mirnov, TRINITI, Troitsk, Russian Federation, Russia

Dr. V.S. Strelkov, Kurchatov Institute, Russian Federation, Russia

Professor Peter Lukac, Katedra Fyziky Plazmy MFF UK, Mlynska dolina F-2, Komenskeho Univerzita, SK-842 15 Bratislava, Slovakia

Dr. G.S. Lee, Korea Basic Science Institute, South Korea

Institute for Plasma Research, University of Maryland, USA

Librarian, Fusion Energy Division, Oak Ridge National Laboratory, USA

Librarian, Institute of Fusion Studies, University of Texas, USA

Librarian, Magnetic Fusion Program, Lawrence Livermore National Laboratory, USA

Library, General Atomics, USA

Plasma Physics Group, Fusion Energy Research Program, University of California at San Diego, USA

Plasma Physics Library, Columbia University, USA

Alkesh Punjabi, Center for Fusion Research and Training, Hampton University, USA

Dr. W.M. Stacey, Fusion Research Center, Georgia Institute of Technology, USA

Dr. John Willis, U.S. Department of Energy, Office of Fusion Energy Sciences, USA

Mr. Paul H. Wright, Indianapolis, Indiana, USA 
The Princeton Plasma Physics Laboratory is operated by Princeton University under contract with the U.S. Department of Energy.

\author{
Information Services \\ Princeton Plasma Physics Laboratory \\ P.O. Box 451 \\ Princeton, NJ 08543
}

Phone: 609-243-2750

Fax: 609-243-2751

e-mail: pppl_info@pppl.gov

Internet Address: http://www.pppl.gov 